\title{
Lusioersily
}

\section{Measurement-Based Path Loss and Delay Spread Propagation Models in VHF/UHF Bands for loT Communications}

Mohamed, E. B., Pugh, J., Brown, C., \& Yanikomeroglu, H. (2018). Measurement-Based Path Loss and Delay Spread Propagation Models in VHF/UHF Bands for IoT Communications. 2017 IEEE 86th Vehicular Technology Conference (VTC-Fall). https://doi.org/10.1109/VTCFall.2017.8287901

Link to publication record in Ulster University Research Portal

Published in:

2017 IEEE 86th Vehicular Technology Conference (VTC-Fall)

Publication Status:

Published (in print/issue): 12/02/2018

DOI:

10.1109/VTCFall.2017.8287901

\section{Document Version}

Author Accepted version

\section{General rights}

Copyright for the publications made accessible via Ulster University's Research Portal is retained by the author(s) and / or other copyright owners and it is a condition of accessing these publications that users recognise and abide by the legal requirements associated with these rights.

\section{Take down policy}

The Research Portal is Ulster University's institutional repository that provides access to Ulster's research outputs. Every effort has been made to ensure that content in the Research Portal does not infringe any person's rights, or applicable UK laws. If you discover content in the Research Portal that you believe breaches copyright or violates any law, please contact pure-support@ulster.ac.uk. 


\title{
Measurement-Based Path Loss and Delay Spread Propagation Models in VHF/UHF Bands for IoT Communications
}

\author{
Ebrahim Bedeer, Jeff Pugh $\dagger$, Colin Brown $\dagger$, and Halim Yanikomeroglu \\ Department of Systems and Computer Engineering, Carleton University, Ottawa, ON, Canada \\ $†$ Communications Research Centre, Box 11490 Station H, Ottawa, Ontario K2H 8S2, Canada \\ Email: \{e.bedeer, halim.yanikomeroglu\}@sce.carleton.ca, \{jeff.pugh, colin.brown\}@canada.ca
}

\begin{abstract}
Internet of Things (IoT) holds a great promise in providing autonomous and ubiquitous connectivity between devices in future communication systems. Due to the spectrum scarcity, very high frequency (VHF) and ultra high frequency (UHF) bands are viewed as valuable resources for IoT communications, especially to connect to distant locations that are hard to reach using higher frequencies. Existing propagation models in the $\mathrm{VHF} / \mathrm{UHF}$ frequency bands are mainly for broadcasting and cellular systems with high transmit antenna heights, and hence, they are not suitable for IoT communications characterized by low antenna heights at both the transmitter and receiver. In this paper, we present new statistical path loss and delay spread models for IoT communications based on quasi-simultaneous wideband channel measurements conducted in the VHF/UHF frequency bands (from 37.8 to $370 \mathrm{MHz}$ ) at the city of Halifax, Canada. In particular, we present two log-distance path loss models (frequency-independent path loss exponent and frequencydependent path loss exponent), as well as, a new statistical distribution of the delay spread.
\end{abstract}

\section{INTRODUCTION}

With the advent of new technologies, it is envisaged that a series of communications will exist between devices, such as cars, sensors, health care gadgets, etc. This paradigm shift in communication had led to the concept of Internet of Things (IoT). Recent studies predicted that the number of connected devices to the Internet will be four times as high as the global population by 2020 [1]. A major challenge for such device-to-device (D2D) communications is the lack of sufficient spectrum [2]. One possible solution to the spectrum scarcity problem is to operate the IoT communications in the unlicensed bands; however, unlicensed bands suffer from excessive interference that will impact/deteriorate the expected performance of D2D communications. Hence, there is a need to investigate alternate spectrum opportunities for IoT communications such as the very high frequency (VHF) and ultra high frequency (UHF) bands of the TV white spaces [3]. This is indeed true if D2D communications are to be reliably used over increasingly greater distances which cannot be supported using higher frequencies.

To design efficient D2D communication systems that operate in the VHF/UHF frequency bands, it is crucial to characterize the wireless propagation channels for such type of communications. Existing propagation models in the VHF/UHF frequency bands were mainly done for broadcast and cellular systems, where the transmit antenna is located at high heights (minimum $30 \mathrm{~m}$ ) and operates at minimum carrier frequencies of $150 \mathrm{MHz}$ [4]. D2D and tactical communications are characterized by low antenna heights for both the transmitter and the receiver and can operate at as low frequencies as $30 \mathrm{MHz}$ [3]. Towards characterizing the wireless propagation channel for low antenna height communications, the authors in [5] proposed a path loss model (with frequency-independent exponent) for military communications operating in the VHF frequency band (30 to $88 \mathrm{MHz}$ ). The proposed model was based on measurements in rural areas in the city of Ottawa, Canada. Path loss exponent values between 3.0 and 3.6 and shadowing standard deviation values between 3.3 and $4.6 \mathrm{~dB}$ were reported, which reflects the prevalence of quasi-line-ofsight (LoS) channel conditions in a flat, rural environment. Measurements were conducted in [6] for tactical ground-toground communications (characterized by low antenna heights at both the transmitter and receiver) for urban environment at the campus of the Johns Hopkins University Applied Physics Laboratory in the VHF band (30-88 MHz). The authors had reported path loss exponent values between 1.5 and 4 and shadow fading standard deviation between 7 and $14.8 \mathrm{~dB}$, which reflect the non line-of-sight (NLOS) propagation in such urban environment. A new delay spread distribution was proposed in [7] for D2D communications operating in the VHF/UHF frequency bands, based on measurements at different environments at eleven locations in Germany.

In this paper, our purpose is to make the extensive data obtained from a measurement campaign held in city of Halifax (hilly and littoral environment), Canada, accessible for the design of tactical and future IoT communication systems operating in the VHF/UHF bands. That said, we present two new propagation models for the log-distance path loss (frequency-independent path loss exponent and frequencydependent path loss exponent). Using a least square fitting, path loss exponents between 4.13 and 4.80 and shadow fading standard deviations between 8.87 and $10.96 \mathrm{~dB}$ are reported. Additionally, a log-normal distribution of the delay spread is proposed with median values between 0.27 and $0.76 \mu \mathrm{sec}$ and standard deviation values between 0.1 and $1.72 \mathrm{~dB}$. 


\section{Measurements And Procedure}

\section{A. Measurements Setup}

The measurement environment in Halifax is characterized by a primary terrain feature that can be described as an upward slope as we move away from the shoreline, and also a corresponding downward slope once we get over the crest of the hill. Many of the measurements were taken beyond the crest of the hill, leading to (sometimes) severe NLoS conditions. While the transmit antenna was at a fixed location, the receiving antenna was mounted on a top of a moving van surveying areas that are populated with residential housing and low-rise buildings. Some measurements were also collected in the city centre. Thus, the terrain and building layouts offered a variety of propagation conditions ranging from lightly obstructed LOS to heavily obstructed NLOS.

The stationary transmit antenna, with a multi-band channel sounder, was located in a research barge in the Birch Cove area of the Bedford Basin. The antenna was mounted at a low height of approximately $10 \mathrm{~m}$ above sea level, while facing Halifax and neighbouring city of Dartmouth. Six carrier frequencies $f$ were selected from the VHF/UHF frequency bands used for D2D and military communications. In particular, $f=37.8,57.0$, and $77.5 \mathrm{MHz}$ are used from the VHF band, while $f=247.25,312.0$, and $370.0 \mathrm{MHz}$ are used from the UHF band. Sounding signals on each centre frequency were simultaneously binary phase shift keying (BPSK) modulated by a 511 bit chip maximal length sequence, combined, amplified, and transmitted from a dual-band, dual-feed whip antenna. The transmit power applied on each carrier frequency of the VHF and UHF frequency bands was 34 and $40 \mathrm{dBm}$, respectively.

A mobile van with roof-mounted antenna was driven around various locations of Halifax and Dartmouth within a 0.5 to 10 $\mathrm{km}$ range of the transmitter. The six frequency bands are recovered from the composite received signal by bandpass filtering and radio frequency (RF) switching. The receiver operates on a quasi-simultaneous manner where sequential recordings of the individual frequency bands over time were performed. An FPGA controls a set of RF switches that select the appropriate front-end signal path and local oscillator for down-conversion. After additional filtering and IQ demodulation, the complex baseband signal is sampled by a high speed A/D card and logged to disk for offline processing.

The measurements reported in this paper were obtained when the receiver operated in a time-triggered mode, i.e., snapshots of the received signal in each frequency band are captured at periodic time intervals. Each snapshot is subsequently converted to a channel impulse response estimate. The aggregate snapshot rate is $350 \mathrm{~Hz}(50 \mathrm{~Hz}$ per each frequency band plus a dead band for validation purposes). The baseband received signal was sampled at a rate of $10 \mathrm{Msamp} / \mathrm{sec}$ (2.857 samples per bit for bit rate of 3.5 Mbps for the $247.25 \mathrm{MHz}$ or 2 samples per bit for bit rate of $5 \mathrm{Mbps}$ for the rest of the frequency bands). Compensation of the measurement system gains and phase shifts was based on a set of calibration

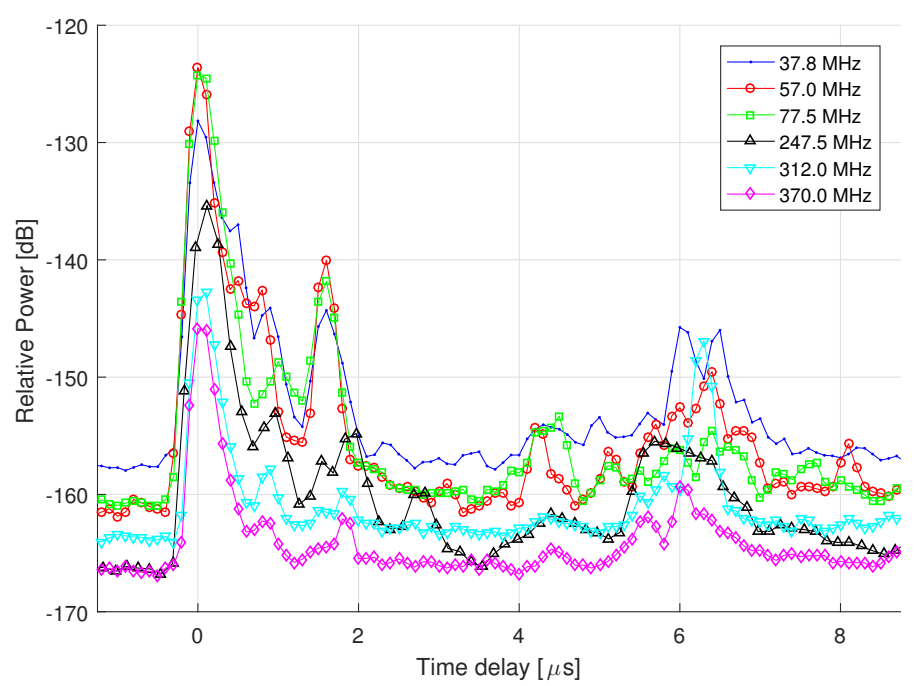

Fig. 1: Average power delay profiles (APDPs) in a residential area at $2.8 \mathrm{~km}$ distance from the transmitter. The profiles are indicative of a strong initial arrival on the direct Tx-Rx path followed by some weaker delayed multipath.

measurements taken with a direct connection between the Tx and Rx units.

\section{B. Data Processing}

The average power delay profile (APDP), $P(\tau)$, is found by averaging impulse responses over short intervals (approximately $10 \mathrm{~m}$ ) along the measurements route. To minimize the influence of noise and interference, components of the APDP which are less than $8 \mathrm{~dB}$ above the median value of the APDP were excluded from our analysis. An example of APDPs in a residential area at $2.8 \mathrm{~km}$ distance from the transmitter is depicted in Fig. 1.

In order to develop general design guidelines that characterize the time dispersive nature of military and IoT communication systems in the VHF/UHF frequency bands, we calculate some parameters such as the mean excess delay and rootmean square (rms) delay spread from the APDP. The rms delay spread can be calculated accordingly as [4]

$$
\tau_{\text {rms }}=\sqrt{\overline{\tau^{2}}-\bar{\tau}^{2}}
$$

where $\bar{\tau}$ is the mean excess delay defined as

$$
\bar{\tau}=\frac{\sum_{k} \tau_{k} P\left(\tau_{k}\right)}{\sum_{k} P\left(\tau_{k}\right)}
$$

and $\overline{\tau^{2}}$ is given as

$$
\overline{\tau^{2}}=\frac{\sum_{k} \tau_{k}^{2} P\left(\tau_{k}\right)}{\sum_{k} P\left(\tau_{k}\right)} .
$$

Similarly, for estimating the parameters of the log-distance path loss models, we calculated the local average power from the recorded impulse responses in order to reduce the influence of the small-scale or multipath fading. As a result the received power can be calculated as the sum of the coefficients of the APDP. Accordingly, the path loss can be calculated as

$$
L=P_{t}+G_{t}+G_{r}-P_{r},
$$




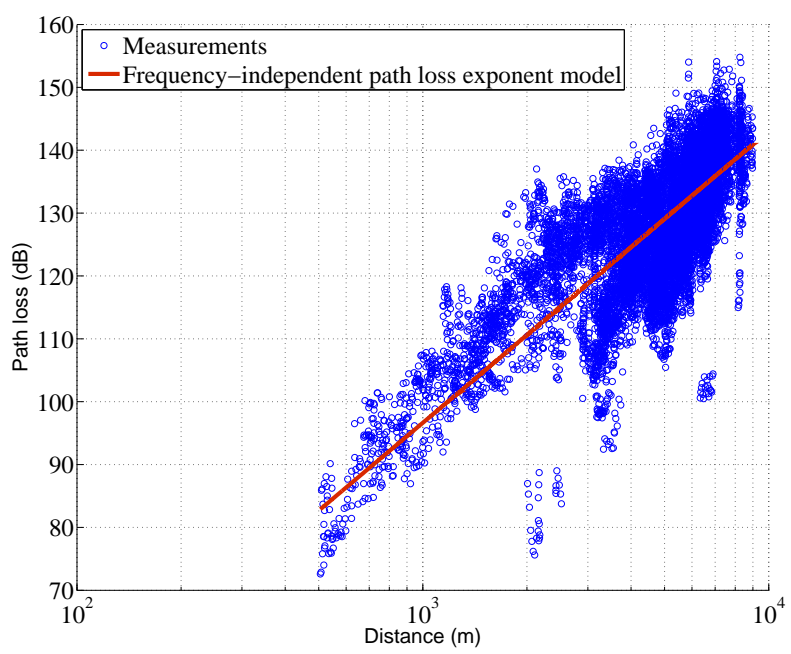

Fig. 2: Estimated frequency-independent path loss exponent model as a function of distance for $f=77.5 \mathrm{MHz}$.

where $P_{t}$ and $P_{r}$ are the transmit and receive powers (in $\mathrm{dBm}$ ), respectively, and $G_{t}$ and $G_{r}$ are the gains (in $\mathrm{dBi}$ ) of the transmit and the receive antennas, respectively. In this paper, $G_{t}=G_{r}=1 \mathrm{dBi}$ are considered in the analysis.

\section{PATH Loss Modeling AND Discussion}

In this section, we present two log-distance path loss models, i.e., frequency-independent path loss exponent and frequency-dependent path loss exponent, for IoT communications operating in the VHF/UHF frequency bands.

\section{A. Frequency-Independent Path Loss Exponent}

In general, the path loss shows a (linear) increasing trend with the (logarithm of) separation between the transmitter and the receiver. Thus, it is common practice to represent the path loss (in $\mathrm{dB}$ ) as the sum of its distance-dependent mean value and a random number that represents shadowing [4]. The path loss $L(d)$ can be written as [4]

$$
L(d)=L_{f s}\left(d_{0}, f\right)+10 \eta \log _{10}\left(d / d_{0}\right)+S, \quad \forall d \geq d_{0},
$$

where $L_{f s}\left(d_{0}\right)$ represents the free space path loss at a reference distance $d_{0}=100 \mathrm{~m}$ and a frequency $f, d$ is the distance between the transmitter and receiver, $\eta$ is the frequencyindependent path loss exponent and $S$ represents the shadow fading variation about the path loss mean value. For each of the six VHF/UHF frequency bands, the log-distance path loss model in (5) is fitted to the local mean path loss values in (4) calculated from the measurements using a least square approach.

Fig. 2 shows a scatter plot of the estimated path loss as a function of the distance between the transmitter and receiver at $f=77.5 \mathrm{MHz}$. As can be seen, the mean value of the path loss is a straight line with respect to the logarithm of the separation between the transmitter and receiver. Additionally, the frequency-independent path loss exponent $\eta$ can be calculated from the slope of the line as 4.64. The measurement deviations

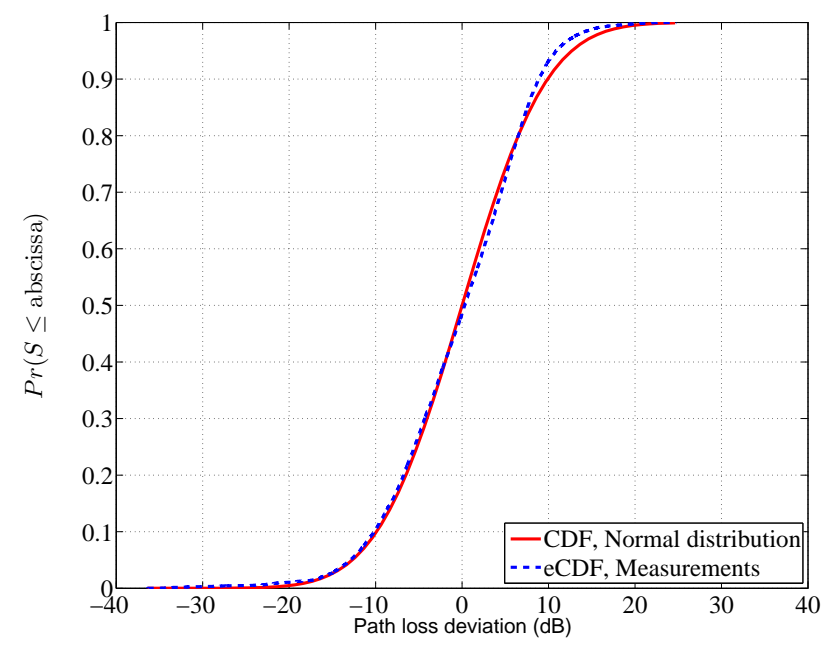

Fig. 3: Shadow empirical distribution and best fit Gaussian distribution for $f=77.5 \mathrm{MHz}$.

around the linear regression line shows large variations; this will be captured in the shadowing modeling as it will be shown in Fig. 3. It is worthy to note that similar results are obtained for the other VHF/UHF frequency bands; however, individual fitting figures are not included due to space limitations. The values of the frequency-independent path loss exponents for all considered VHF/UHF frequency bands are presented and discussed later in Fig. 4.

Fig. 3 compares the empirical cumulative distribution function (eCDF) of the shadow fading of the log-distance path loss model in (5) at $f=77.5 \mathrm{MHz}$ with its best Gaussian CDF fit. The mean and standard deviation of the best Gaussian fit were obtained by a maximum likelihood estimation and were found to be 0 and $8.86 \mathrm{~dB}$, respectively. One can notice that the high standard deviation value, when compared to its counterpart in similar studies in [5], reflects the difference between the two measurements environments. In particular, while the measurements in [5] were done for rural environment characterized by LOS, the measurements reported in this paper are for hilly and littoral environment mainly characterized by a wider range of propagation conditions, i.e. LoS and heavy NLoS. One can additionally notice from Fig. 3 that there is a good match between the eCDF and its best Gaussian fit. To quantify how good is the fit, goodness-of-fit by means of the Kolmogorov-Smirnov (K-S) test [8] was carried out on the eCDF and the log-normal CDF. Numerical results of the $\mathrm{K}-\mathrm{S}$ test rejects the null hypothesis at the $5 \%$ significance level. Hence, we concluded that log-normal distribution is an acceptable measure for the shadow fading statistics (as expected). Similar results are obtained for the other VHF/UHF frequency bands; however, individual fitting figures are not included due to space limitations. The values of the standard deviation values for all considered VHF/UHF frequency bands are presented and discussed later in Fig. 5. 


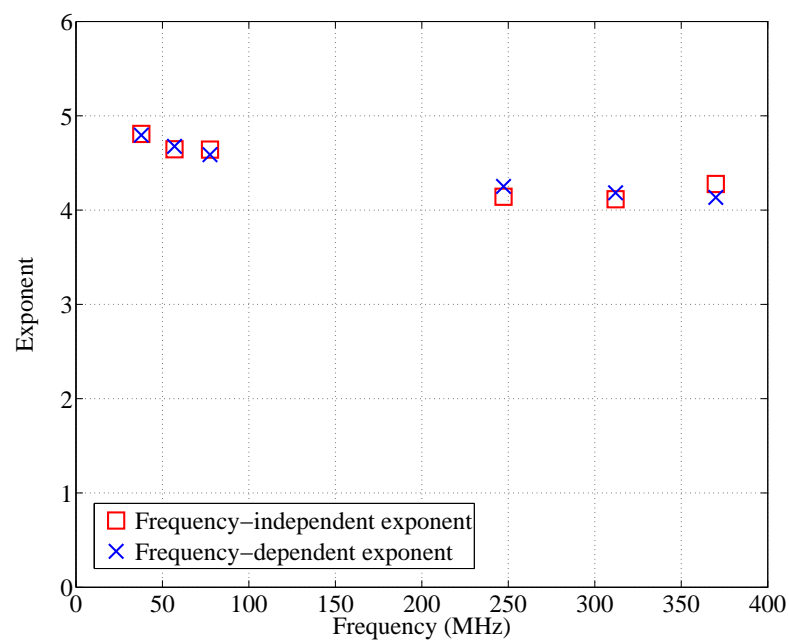

Fig. 4: Estimation of frequency-independent path loss exponent $\eta$ and frequency-dependent path loss exponent $\eta(f)$ for different frequencies in VHF/UHF bands.

\section{B. Frequency-Dependent Path Loss Exponent}

In this model, the path loss exponent is determined jointly over all the six considered frequency bands. That said, the log-distance path loss model can be written as

$$
\begin{array}{r}
L(d, f)=L_{f s}\left(d_{0}, f\right)+10 \eta(f) \log _{10}\left(d / d_{0}\right)+S, \\
f \geq 30 \mathrm{MHz}, \forall d \geq d_{0},
\end{array}
$$

where $\eta(f)$ is the frequency-dependent path loss exponent that is given by

$$
\eta(f)=\eta_{0}+K_{\eta} \log _{10}\left(f / 30 \times 10^{6}\right) .
$$

The term $f / 30 \times 10^{6}$ gives the frequency in $\mathrm{MHz}$ such that $\eta_{0}$ is the $30 \mathrm{MHz}$ intercept of the frequency-dependent path loss exponent. $K_{\eta}$ represents the slope of the exponent versus the logarithm of the normalized frequency. The results of estimating the frequency-dependent path loss exponent using a least square approach are $\eta_{0}=4.86$ and $K_{\eta}=-0.667$.

Fig. 4 compares the path loss exponent from both logdistance pass loss models in (5) and (6). As can be seen, both models results in a similar estimation of the path loss exponent. One can conclude that the path loss exponent for such type of environment (i.e., littoral and hilly) ranges between 4.81 and 4.28 for the considered frequencies in the VHF/UHF bands. It is worthy to mention that the average fitting errors of the frequency-dependent path loss model did not increase significantly when compared to their counterparts of the frequency-independent path loss model.

Fig. 5 plots the shadow fading standard deviation for both log-distance path loss models in (5) and (6). As in the discussion on Fig. 4, similar standard deviation values are obtained for both models. This confirms that either model can be used for designing future IoT communication systems. We also note that the standard deviation value increases with increasing the centre frequency.

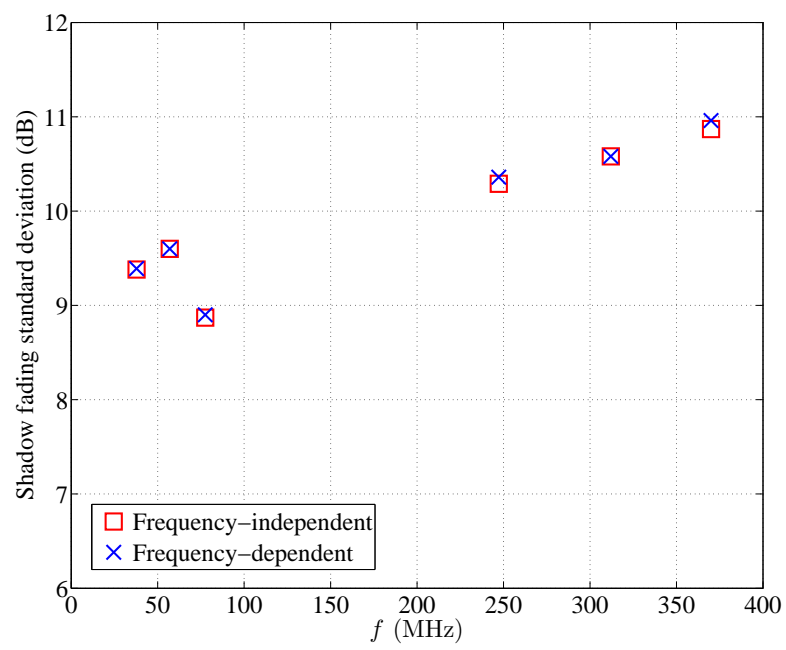

Fig. 5: Estimation of frequency-independent and frequency-dependent shadow fading standard deviation (in $\mathrm{dB}$ ) for different frequencies in VHF/UHF bands.

To characterize how similar the wireless channel propagation over the six VHF/UHF frequency bands, Table I summarizes the shadow fading correlation coefficients. As can be seen, the correlation coefficients are in general high which reflect the very similar propagation characteristics over different VHF/UHF frequency bands for IoT communications. This is especially the case for the UHF band, where the shadow fading correlation coefficients are close to 1 (i.e., 0.97 and $0.98)$.

\section{Delay Spread Modeling and Discussion}

It is known that the empirical probability density function of the delay spread $\tau_{\text {rms }}$ follows a log-normal distribution [4]. We used the maximum likelihood estimation in order to fit the calculated $\tau_{\text {rms }}$ from the measurements to the log-normal distribution.

In Fig. 6, we compare the eCDF of the delay spread $\tau_{\text {rms }}$ at $f=370.0 \mathrm{MHz}$ and the CDF of a log-normal distribution with median and standard deviation values of $\tau_{\mathrm{med}}=0.71 \mu \mathrm{sec}$ and $\sigma_{\tau_{\mathrm{rms}}}=1.72 \mathrm{~dB}$, respectively. As can be seen, both curves are in a good agreement. K-S test are performed to numerically quantify the goodness-of-fit, and the null hypothesis at $5 \%$ significance level was rejected. Similar results are obtained for other frequencies; however, individual figures are not included due to space limitations.

Fig. 7 shows the median value of the delay spread obtained from measurements for different VHF/UHF frequency bands,

TABLE I: Shadow fading cross-correlation coefficients

\begin{tabular}{|r|l|l|l|l|l|l|}
\hline$f(\mathrm{MHz})$ & 37.8 & 57 & 77.5 & 247.25 & 312 & 370 \\
\hline 37.8 & 1.00 & 0.87 & 0.84 & 0.85 & 0.84 & 0.84 \\
\hline 57 & 0.87 & 1.00 & 0.88 & 0.84 & 0.83 & 0.82 \\
\hline 77.5 & 0.84 & 0.88 & 1.00 & 0.91 & 0.90 & 0.89 \\
\hline 247.25 & 0.85 & 0.84 & 0.91 & 1.00 & 0.97 & 0.97 \\
\hline 312 & 0.84 & 0.83 & 0.90 & 0.97 & 1.00 & 0.98 \\
\hline 370 & 0.84 & 0.82 & 0.89 & 0.97 & 0.98 & 1.00 \\
\hline
\end{tabular}




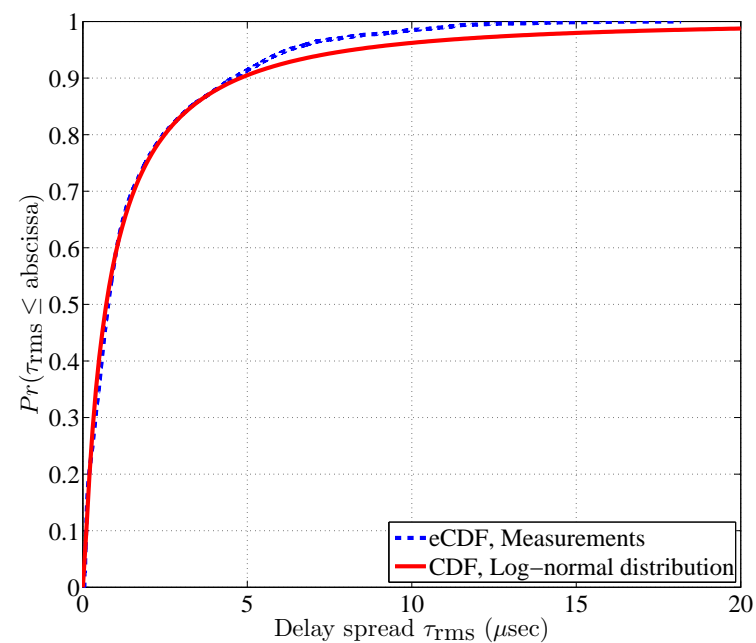

Fig. 6: Empirical CDF of the delay spread $\tau_{\text {rms }}$ at $f=370.0 \mathrm{MHz}$ (dashed) and CDF of log-normal distribution (solid) with $\tau_{\text {med }}=$ $0.71 \mu \mathrm{sec}$ and $\sigma_{\tau_{\mathrm{rms}}}=1.72 \mathrm{~dB}$.

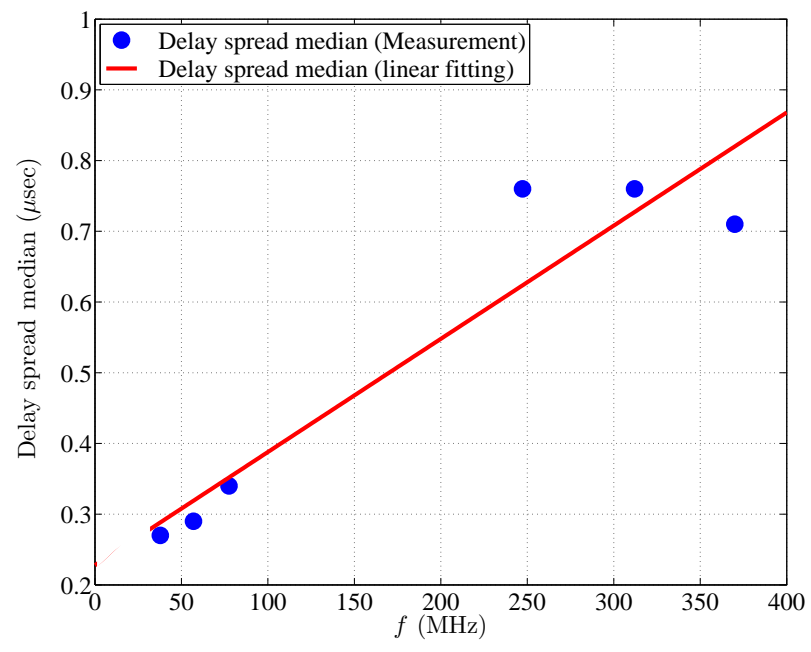

Fig. 7: Median of delay spread $\tau_{\text {med }}$ for different frequencies and the fitted delay spread line

in addition to a linear fitting line to the median values. One can see that the delay spread median value increases with increasing the frequency, and its value in the VHF band ranges between 0.25 to $0.35 \mu \mathrm{sec}$ and in the UHF band ranges between 0.7 and $0.75 \mu$ sec.

Fig. 8 depicts the standard deviation value of the delay spread obtained from measurements for different VHF/UHF bands, in addition to a linear fitting line. As can be seen, the standard deviation of the delay spread in such environment increases with frequency and can reach up to $1.72 \mathrm{~dB}$.

\section{CONClusion}

In this paper, we presented new statistical log-distance path loss and delay spread models for IoT communications operating in the VHF/UHF frequency bands (37.8 to $370 \mathrm{MHz}$ ). These models are based on quasi-simultaneous wideband

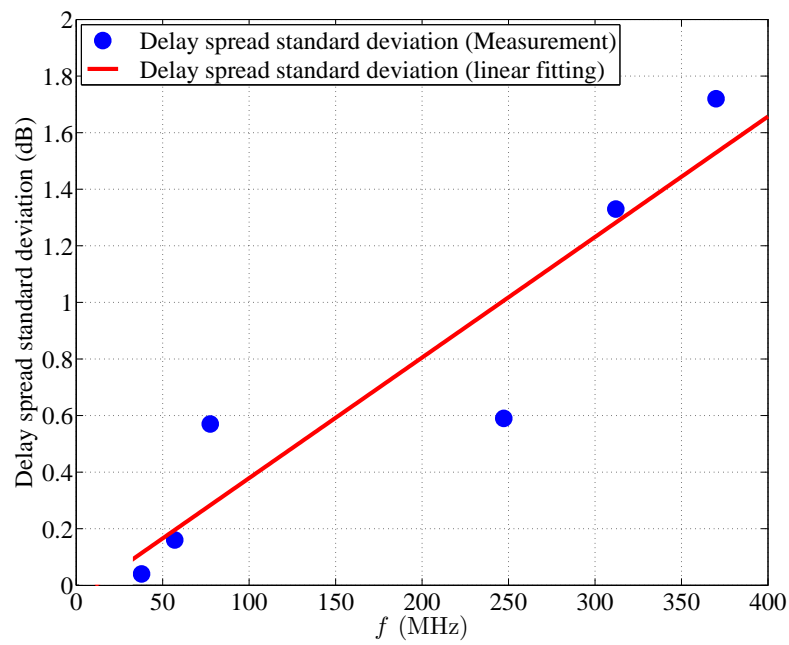

Fig. 8: Standard deviation of delay spread $\sigma_{\tau_{\text {med }}}$ for different frequencies and the fitted delay spread line

channel measurement campaign held in the city of Halifax, Canada. We noticed that both path loss models (frequencyindependent and frequency-dependent exponent) give similar estimation results. In particular, for this type of environment, high values of the path loss exponent (between 4.13 and 4.80) are reported. Additionally, standard deviation values of the shadow fading are in the range of 8.87 and $10.96 \mathrm{~dB}$. Additionally, we showed that the rms delay spread can be modeled as a log-normal distribution with increasing median and standard deviation values with increasing the operating frequency.

\section{ACKNOWLEDGMENT}

The work was supported by Defence Research and Development Canada (DRDC).

\section{REFERENCES}

[1] D. Lake, A. Rayes, and M. Morrow, "The internet of things," The Internet Protocol Journal, vol. 15, no. 3, pp. 10-19, Sep. 2012.

[2] E. Bedeer, M. Marey, O. Dobre, and K. Baddour, "On partially overlapping coexistence for dynamic spectrum access in cognitive radio," in Proc. IEEE CAMAD, Jun. 2011, pp. 143-147.

[3] Ofcom, "TV white spaces: approach to co-existence," Sep. 2013, [Accessed Mar. 28, 2017]. [Online]. Available: http://stakeholders.ofcom.org.uk/binaries/consultations/white-spacecoexistence/annexes/technical-report.pdf

[4] T. Rappaport, Wireless Communications: Principles and Practice. Prentice Hall PTR New Jersey, 1996.

[5] J. A. Pugh, R. J. C. Bultitude, and P. J. Vigneron, "Path loss measurements with low antennas for segmented wideband communications at VHF," in Proc. IEEE Military Communications Conference, Oct. 2006, pp. 1-5.

[6] J. Andrusenko, R. L. Miller, J. A. Abrahamson, N. M. M. Emanuelli, R. S. Pattay, and R. M. Shuford, "VHF general urban path loss model for short range ground-to-ground communications," IEEE Trans. Antennas Propag., vol. 56, no. 10, pp. 3302-3310, Oct. 2008.

[7] J. Fischer, M. Grossmann, W. Felber, M. Landmann, and A. Heuberger, "A novel delay spread distribution model for VHF and UHF mobileto-mobile channels," in Proc. European Conference on Antennas and Propagation, Apr. 2013, pp. 469-472.

[8] F. J. Massey Jr, "The Kolmogorov-Smirnov test for goodness of fit," Journal of the American statistical Association, vol. 46, no. 253, pp. 6878, Mar. 1951. 